\title{
El e-commerce y las Mipymes en tiempos de Covid-19
}

\section{E-commerce and MSMEs in times of Covid-19}

\author{
RODRÍGUEZ, Kelly G. ${ }^{1}$ \\ ORTIZ, Olga J. ${ }^{2}$ \\ QUIROZ, Alicia I. ${ }^{3}$ \\ PARRALES, María L. ${ }^{4}$
}

\section{Resumen}

El Covid-19 ha desafiado al sistema socioeconómico mundial y las Mipymes ecuatorianas, afectadas por la paralización de sus actividades productivas debido a la contracción de la demanda por confinamiento social, se han visto en la necesidad de repensar sus modelos de negocio. Analizar el impacto del Ecommerce en las Mipymes por la disrupción de la pandemia bajo un enfoque documental, permitió conocer que el comercio tradicional pasó a ser una estrategia de segundo plano, y el comercio electrónico una de las industrias ganadoras.

Palabras clave: comercio electrónico, covid-19, mipymes, resiliencia

\begin{abstract}
Covid-19 has challenged the world socio-economic system and Ecuadorian MSMEs, affected by the paralysis of their productive activities due to the contraction of demand due to social confinement, have found it necessary to rethink their business models. Analyzing the impact of E-commerce on MSMEs due to the disruption of the pandemic under a documentary approach, will get to know that traditional commerce became a background strategy, and electronic commerce one of the winning industries.

Key Words: electronic commerce, covid-19, MSMEs, resilience
\end{abstract}

\section{Introducción}

La realidad a la que estábamos acostumbrados a dado un giro de $360^{\circ}$ grados, la emergencia mundial generada por el Covid-19 ha marcado un punto de inflexión y un desafío para la humanidad; la manera de educarse, de trabajar, de entretenerse, y, sobre todo de comunicarse ha cambiado. En tal sentido, una de las alteraciones más grandes que se ha observado, es la forma en que se hacen negocios. Ahora, estar conectado cobra más relevancia que nunca, avanzar hacia la digitalización es imperante.

La globalización y los avances vertiginosos de la tecnología han provocado que los mercados se comporten, cada vez, de una manera diferente. Con la aparición del internet muchas de las formas tradicionales de negociación,

\footnotetext{
1 Estudiante Administración de empresas. Facultad de ciencias económicas, Universidad Estatal del Sur de Manabí, Ecuador. Email: rodriguezkelly4560@unesum.edu.ec

2 Estudiante Administración de empresas. Facultad de ciencias económicas, Universidad Estatal del Sur de Manabí, Ecuador. Email: ortizolga6342@unesum.edu.ec

3 Estudiante Administración de empresas. Facultad de ciencias económicas, Universidad Estatal del Sur de Manabí, Ecuador. Email: quirozalicia8463@unesum.edu.ec

${ }^{4}$ Economista, Magister en Administración de Empresas, Doctora en Ciencias Pedagógicas. Facultad de Ciencias Administrativas y Económicas, Universidad Estatal del Sur de Manabí, Ecuador. Email: maria.parrales@unesum.edu.ec
} 
compra y venta, se han transformado. En Ecuador el 82,3\% de las Mipymes utilizan la internet. La proporción de uso es la siguiente: microempresas $48,6 \%$, medianas $56,9 \%$ y pequeñas $52,9 \%$, dando un total general de $52,8 \%$. Sin embargo, a pesar del acceso que tienen a internet se limitan a usarlo para enviar correos y realizar funciones administrativas, solo un 27,4\% de las Mipymes tiene presencia en la web. (Ministerio de Telecomunicaciones y de la Sociedad de la Información, 2020)

Es así como, la situación actual producto del Covid-19 está forzando a muchas empresas a repensar sus modelos de negocio; la nueva realidad está obligando a realizar compras habituales a través de transacciones electrónicas, por lo que para las Mipymes algunos de los mecanismos tradicionales de mercado podrían no ser suficientes para enfrentarla debido a que la digitalización se ha vuelto un requisito obligatorio a nivel empresarial.

Los negocios tradicionales, enfrentan nuevos retos para comercializar su producción, por lo que, sin duda, aquellas que son capaces de afrontar desafíos, generando cambios y avanzando hacia lo digital pueden adaptarse al escenario comercial del e-commerce. Este método de compra-venta por internet permite a los gerentes entrar en la nueva era del comercio para que puedan cruzar fronteras, mejorando y agilizando sus procesos y en efecto crecer en el mercado. Todo esto, es parte de la innovación de las empresas, Schumpeter (1934) apostaba por la innovación, como un proceso de ejecutar nuevas combinaciones, cuando se establecen nuevas combinaciones se generan nuevas formas de cubrir la demanda existente o crear nuevos productos, a menudo generando la obsolescencia de las tecnologías, productos y procesos actuales, a esto Schumpeter lo consideró un proceso de destrucción creativa.

Por ello, los autores de la investigación, buscan analizar el impacto del e-commerce en las Mipymes ecuatorianas por la disrupción de la pandemia del Covid-19; es así como el comercio electrónico es la opción más segura para adquirir bienes sin tener que salir de casa. Para sobrevivir en el actual contexto de impactos causados por la pandemia, muchas empresas tienen que recurrir al uso intensivo de herramientas digitales para implementar el teletrabajo, realizar compras y ventas online, así como gestionar procesos de producción de forma remota.

La transformación digital, que antes parecía un extra para aumentar la productividad y las ganancias hoy se ha vuelto un requisito para que las empresas sobrevivan. En particular, las Mipymes que comienzan a transitar por la ruta de la transformación digital, necesitan dotarse muy rápidamente de una serie de elementos, aquellas que comiencen a utilizar las plataformas digitales y tengan estrategias que las acompañen son aquellas que pueden tener más facilidad para reorientar sus empresas y mantenerse en el mercado.

Es de vital importancia que las Mipymes ecuatorianas se adapten al e-commerce, visto hoy como la nueva metodología para comercializar sus productos, en el momento en que nos encontramos y en un futuro cercano. Los investigadores, recurren a fuentes documentales para recopilar conceptos y opiniones importantes de otros investigadores y fundamentar la investigación que apoyada en los métodos lógicos y empíricos permitió observar la realidad y experiencia social en un momento en donde lo habitual pasó a un segundo plano. Se presentaron algunas limitaciones debido a la escasez de información y estadísticas en el uso del comercio electrónico por las Mipymes en tiempos de Covid-19.

La pandemia superó uno de los retos dentro de la industria en Ecuador, la digitalización en los negocios, las Mipymes lograron sacar esa capacidad de adaptación, y ahora muchas utilizan el e-commerce para no perder su mercado, aun así, la consolidación de los comercios electrónicos señala un camino medianamente explorado en Ecuador y que se espera en el mediano plazo aumente. Para alcanzar el éxito, los negocios deben de enmarcarse dentro de la versatilidad empresarial para responder a la exigencia de cambios que dependen mucho de algunos factores importantes como la disponibilidad de herramientas tecnológicas, la sinergia desarrollada en los equipos de trabajo y la discusión al cambio en su cultura organizacional. Ademas, las políticas públicas que decrete el gobierno de Lenin Moreno Garcés, son fundamentales para reactivar la economía de las Mipymes. 
Para cumplir con el objetivo propuesto, este artículo se organiza en varios epígrafes enfocados en las Mipymes en Ecuador; el e-commerce en Ecuador y el panorama en Sudamérica; las plataformas del e-commerce e instituciones vinculadas a su desarrollo en Ecuador; se explica lo que ofrece el comercio electrónico a las Mipymes, abordando el crecimiento del e-commerce durante la pandemia del Covid-19 y el impacto a nivel nacional; finalmente, se presenta el diseño metodológico, los resultados, la discusión, las conclusiones y recomendaciones.

\subsection{Desarrollo}

Actualmente el mundo vive una emergencia sanitaria, humana y económica sin precedentes. Ante la enfermedad por coronavirus, acrónimo del inglés coronavirus disease 2019, también conocida como enfermedad por coronavirus en español, las economías se cierran y paralizan, y las sociedades entran en cuarentenas más o menos severas, la restricción de movilidad a medida que se extiende, hace que el ser humano busque desarrollar nuevas rutinas o actividades para recuperar el balance perdido a raíz del aislamiento.

Muchos podrán están incorporando rutinas de ejercicios físicos, hábitos de lectura, escuchando música, viendo películas o simplemente compartiendo con su familia, lo cierto aquí, es que adicional a esto, el Covid-19 ha forzado a realizar compras habituales a través de transacciones electrónicas lo que indica para algunas personas nuevas experiencias en la web y, para otros el incremento de una conducta previamente adquirida.

A nivel mundial la economía se ha visto severamente afectada por el Covid-19, en algunos países se ha interrumpido la producción, ha decrecido la demanda de bienes y servicios, ha puesto en suspenso las cadenas de suministro, obligando a las empresas a suspender o reducir sus operaciones. Las Mipymes son las más vulnerables a estos desafíos, ya que tienen menos activos, son particularmente dependiente de sus transacciones en persona y de reservas de efectivo más condicionadas que las grandes empresas.

El fuerte impacto que acarreó el Covid-19 a la economía ecuatoriana, ha creado en la Mipymes la necesidad urgente de reinventarse, esta crisis ha cambiado la forma en que funcionan estas empresas y ha obligado a pensar a este grupo de agentes económicos que representan el $99.5 \%$ del tejido empresarial (microempresas $90,78 \%$, pequeñas $7,22 \%$ y medianas $1,55 \%$ ), dinamizan la economía con el $60 \%$ de empleo y el $50 \%$ al Producto Interno Bruto (PIB) de acuerdo al Instituto Nacional de Estadísticas y Censos (INEC) cómo comprar, vender y sobre todo funcionar en la nueva realidad exigida por el Covid-19. (Diario El Mercurio, 2020)

Los negocios tradicionales, enfrentan nuevos retos para comercializar su producción. Del comercio directo ahora deben no solo implementar la entrega a domicilio, sino también al e-commerce como sus aliados para mantener el negocio y la nómina. La Comisión Económica para América Latina y el Caribe (CEPAL), enfatiza que la pandemia es inusitada en la medida que plantea un desafío al sistema socio-económico mundial algunos de los mecanismos tradicionales de mercado podrían no ser suficientes para enfrentarla debido a la interrupción de las actividades productivas y la consiguiente contracción de demanda. (CEPAL, 2020)

El Covid-19 ha alterado la forma en que funcionan las empresas, ha impulsado especialmente a las Mipymes a evolucionar sus modelos de negocios, revaluando y replanteando su supervivencia en el mercado primordialmente, la transformación digital se convierte en el camino para lograrlo. Todos los sectores económicos de las Mipymes se vieron afectados, en particular los sectores de la aviación, el turismo y de servicios como el comercio (OIT, 2020). Sin embargo, en este contexto producto de la situación sanitaria y la restricción de movilidad algunas son privilegiadas por la transformación digital, que les permite mantenerse virtualmente operativas y poder continuar sirviendo a sus clientes. El e-commerce destaca como una de las industrias ganadoras. 
Actualmente, el Covid-19 está haciendo que las transacciones comerciales físicas que se realizaban de acuerdo a la realidad a la que se estaba acostumbrado, den un giro y pasen a ser transacciones comerciales electrónicas. Lo que para las Mipymes significa que la transformación digital dejó de ser un complemento para aumentar la productividad y las ganancias, pasando a ser un requisito para sobrevivir.

En tal sentido, los trabajos de Joseph Schumpeter sobre el emprendimiento y la innovación, y su efecto en el desarrollo económico constituyen una de las contribuciones teóricas de mayor importancia en el enfoque de este trabajo. El e-commerce ha hecho que las empresas cambien sus métodos tradicionales de venta, obligando a los gerentes a entrar en la nueva era del comercio para que puedan cruzar fronteras, mejorando y agilizando sus procesos de ventas y en efecto crecer en el mercado. De eso se desprende, considerar a la innovación como un proceso que consiste en poner en práctica inventos previos de cualquier naturaleza o aplicación de la invención a usos industriales y comerciales, señalando que los emprendedores deben de ser innovadores destacando por su perseverancia y su ambición. (Schumpeter, 1934)

Los aportes investigativos de Schumpeter establecen que cualquier individuo es un emprendedor cuando ejecuta nuevas combinaciones. Cuando se establecen nuevas combinaciones se generan nuevas formas de cubrir la demanda existente o crear nuevos productos, a menudo generando la obsolescencia de las tecnologías, productos y procesos actuales, a esto Schumpeter lo consideró un proceso de destrucción creativa. Al respecto los autores Sornoza Parrales, Parrales Poveda, Sornoza Parrales y Cañarte Rodriguez (2018) afirman que:

Los emprendimientos crecen gracias a un proceso dual de tomar la cuota de mercado de los proveedores existentes y de incrementar la demanda general de los productos ofrecidos en el mercado. Por tanto, el proceso de destrucción creativa está basado en esfuerzos dinámicos y deliberados para cambiar las estructuras de los mercados y puede propender a la generación de innovaciones adicionales y oportunidades de rentabilidad. (p.15)

El negocio de Mipymes con el Covid-19 se debilitó fuertemente, muchos negocios han cerrado sus puertas o reducido significativamente sus operaciones, debido a que la pandemia exige un replanteamiento de sus estructuras, las cuarentenas forzosas producen la virtualización de relaciones económicas y sociales, convirtiéndose esto en un reto para el segmento mayoritario de firmas existentes a quienes les cuesta más subirse a esta ola digital.

En este marco se comenzará señalando que no existe una definición ni clasificación universalmente dada para Mipymes, para Rodríguez Valencia (2007) el concepto varía de acuerdo a diferentes criterios, pero considera que la empresa es el principal factor dinámico de la economía de una nación.

La Comunidad Andina de Naciones (CAN) define a las Mipymes como actores centrales en el tejido productivo y social de los países andinos, siendo grandes generadoras de empleo, usuarias de tecnología intensiva en mano de obra y procesando insumos básicamente nacionales. (CAN, s.f.)

Las Mipymes juegan un papel transcendental en la economía ecuatoriana, dinamizan el aparato productivo, por la variedad de bienes y servicios que ofrecen, así como también son generadoras de empleos, convirtiéndose en la base fundamental del tejido social. Por lo que Rodríguez Valencia afirma que: "influyen de manera directa en la vida privada de sus habitantes (trabajo, subsistencia, medio de vida, prosperidad, calidad de vida, entre otras" (2007, p.23).

Estas unidades productivas son regularizadas por diferentes agentes de control como la Superintendencia de Compañías, Seguro Social, Ministerio de Relaciones Laborales, Banco Central, Servicio de Rentas Internas, entre otras". (Granda Bohórquez, 2020, pág. 11) 
Según el clasificador Internacional Industrial Único (CIIU), las actividades en que se involucran son las siguientes: comercio al por mayor y al por menor, agricultura, silvicultura y pesca, industrias manufactureras, construcción, transporte, almacenamiento y comunicaciones, bienes inmuebles y servicios prestados a las empresas, servicios comunales, sociales y personales. (Granda Bohórquez, 2020, pág. 10)

Según el Boletín Juridico de la Cámara de Comercio de Quito (2017) en Ecuador, la empresas se clasifican en base a los preceptos básicos del Programa Estadístico Comunitario de la Comunidad Andina de Naciones (CAN). La Superintendencia de Compañías, Valores y Seguros, mediante resolución, acogió la clasificación de micro, pequeñas, medianas y grandes empresas, de acuerdo con la normativa implantada por la Comunidad Andina en su Resolución 1260 y la legislación interna vigente, conforme a los siguiente:

Microempresa: Personal ocupado 1-9; Valor bruto en ventas menor a 100.000; Monto de activos hasta 100.000

Pequeña Empresa: Personal ocupado 10-49; Valor bruto en ventas igual o mayor a 100.001-1.000.000; Monto de activos de 100.001 hasta 750.000

Mediana empresa: Personal ocupado 50-199; Valor bruto en ventas igual o mayor 1.000.001 - 5.000.00; Monto de activos de 750.001 hasta 3.999.999

La gran mayoría de empresas en Ecuador, pertenecen a la categoría de Mipymes, según el último Censo Nacional Económico del 2010, es así que 9 de cada 10 establecimientos están dentro de esta categoría, lo que ratifica su relevancia en la economía del país. Actualmente se encuentran alrededor de 880.203 Mipymes. Las provincias que albergan la mayoría de las microempresas son Guayas con un 39, 93\% que equivale 14.970 y Pichincha con un 33,53\% que equivale a 12.570. De igual forma estas son las provincias que alojan la mayoría de las Pymes, la provincia del Guayas abarca un total del $32.67 \%$ que representa a un total de 58.574 empresas de este tipo, seguido de la provincia de Pichincha con un aporte del 27.95\% equivalente a una cantidad de 50.269 pymes y por último la provincia de Manabí con un $4.69 \%$ que equivale a 8.438 empresas. (INEC, 2016)

El resto de provincias suman un alto porcentaje de estas empresas, de las cuales la mayoría se dedica al comercio y los servicios; junto con la construcción y la manufactura, estas acaparan un rol fundamental en la economía debido a que son el sector más productivo. En Ecuador, el 39\% de los empleos son generados por microempresas, mientras que el $17 \%$ a pequeñas y $14 \%$ a medianas. ( Ron Amores y Sacoto Castillo, 2017, pág. 6)

La región en donde más Mipymes se concentran es en la Costa con un número de 85.363 empresas de este tipo seguido por la región Sierra, aunque con una diferencia mínima posee un número de 85.069 pymes, el oriente cuenta con 8.839 pymes y la región insular con apenas 559 Pymes. (INEC, 2016)

Las Mipymes son la fuente de movimiento de la economía del Ecuador, con la paralización de las actividades productivas en un $70 \%$ muchas se vieron en la necesidad de buscar respuesta a esta nueva situación para abordar la serie de desafíos económicos planteados por la crisis de Covid-19. La respuesta encontrada es la digitalización. La tecnología está en el centro de la transformación para las Mipymes e incluso en nuestra vida cotidiana.

Las Tecnologías de Información y Comunicación (TIC), indiscutiblemente han contribuido al desarrollo de un fuerte vínculo entre el comercio y la tecnología en general, permitiendo a usuarios y empresas acercarse a un mundo más digitalizado (Piñeiro y Rodríguez, 2016).

El Covid-19 prácticamente obligó a las Mipymes a migrar hacia la virtualización de actividades, convirtiéndose en catalizadores del e-commerce. La capacidad de resiliencia que tiene este grupo de agentes económicos, así como la capacidad de adaptación, les ha permitido introducirse a las nuevas tecnologías, convirtiéndose esto en parte de la evolución de las empresas, siendo un proceso beneficioso para el éxito de cualquier organización. 
Hoy en día los mercados globales reflejan la heterogeneidad cultural, de productos y la globalización de los estilos de vida. El e-commerce está acelerando estas tendencias. Sornoza Parrales, et al. (2018) al respecto mencionan que:

Es por sí misma, la innovación que afecta de mayor manera a los negocios en el siglo XXI y está creando enormes oportunidades para las pequeñas empresas. Los emprendedores de e-commerce explotan las oportunidades inmediatas de mercado para crear estructuras comerciales. Algunas empresas compiten en precio, pero otras usan el e-commerce para diferenciarse más efectivamente y alcanzar nichos de mercado para sus productos o servicios con un alcance global. Por tanto, el número de nichos de mercado aparenta crecer exponencialmente. (p.84)

El comercio electrónico, como se lo conoce mundialmente es la actividad que implica la compra-venta de productos o servicios con la característica de que en este proceso intervienen medios o herramientas tecnológicas, como es el caso de la internet (García, 2018).

Este ha tenido desde sus inicios cuatro generaciones, de acuerdo a Seoane (2005) se detallan a continuación:

Primera Generación: En el año 1993, en las grandes empresas surgió la necesidad e importancia de crear páginas web, pero aún no se podían hacer comparas por la web, solo brindar información para la organización. Simplemente las comunicaciones con el comprador se redujeron a formularios o correos electrónicos.

Segunda Generación: Las empresas empezaron a usar sus páginas para hacer negocios con las personas a través de la red y desde entonces aparecen los centros comerciales virtuales y los usuarios empezaron hacer sus compras por internet. En esta etapa los medios de pago por la adquisición de los bienes y servicios eran con reembolsos, transferencias, cheques y con tarjetas electrónicas.

Tercera Generación: Las empresas quisieron que el proceso de selección y envío de los datos de los productos comprados sea automático, implementando sistemas de bases de datos, con aplicaciones web, basadas en guiones CGI (Common Gateway Interface). Aparece el "marketing en la red" y se generaliza el uso de las tarjetas como medio de pago y se crean protocolos para un pago seguro.

Cuarta generación: El contenido de la información en internet es dinámico, generado por una aplicación web de los datos de un sistema, se emplearon diseñadores gráficos para la creación e informáticos para el soporte y diseño del negocio. Se implementan diferentes mecanismos de pago seguro y se mejora la seguridad de los sitios de la red.

Originada la nueva modalidad de compra-venta por internet tanto países desarrollados como en vías de desarrollo, empezaron a adoptarla. Desde países como EEUU, países de América Latina y Ecuador. Actualmente en Ecuador a raíz de los impactos causados por el Covid-19 el e-commerce, actividad que hasta finales del año pasado crecía a un ritmo lento del $20 \%$ anual, se aceleró desde el mes de abril cuando empezó la emergencia sanitaria. (Zapata, 2020)

En tiempos de Covid-19 el uso de la tecnología se ha vuelto prácticamente un requisito obligatorio a nivel empresarial, más si se trata de actividades comerciales. Si una empresa no se adapta a las tendencias actuales, fácilmente terminaría perdiendo su mercado. Las formas tradicionales de negociación, compra y venta, se han transformado en las empresas debido al buen uso que se le atribuye a la tecnología (Aguerre, 2019). La rapidez y fiabilidad en las operaciones son beneficios del e-commerce, a su vez este cuenta con estrategias asociadas con almacenamiento, reducción de costos lo que permite disminuir la ineficiencia, producir más cantidad, más rápido, de mejor calidad, en menos tiempo y le ayudan a la competitividad. 
Adaptar la Mipymes tecnológicamente puede ser visto como un problema. Pero la dificultad para X, no puede ser la dificultad de $Y$, por lo que, los directivos de las organizaciones, deben centrarse en escoger la estrategia y el modelo que más se adapte a sus necesidades (Parrales Poveda, 2013). Es así que la literatura ofrece infinidad de modelos de comercio electrónico, que a consideración de Tello Pérez y Pineda González (2017) se cita a continuación:

B2B o business to business: la transacción se realiza entre empresas que operan en internet. B2C o business to costumer: es el comercio entre la empresa que produce, la que vende o prestadora de servicios y el consumidor final. B2E o business to employee: los negocios se realizan entre la empresa y sus colaboradores con beneficios exclusivos. C2C o costumer to costumer: transacción en la que el cliente adquiere un producto o servicio y realiza la acción de reventa. G2C o government to costumer: la transacción es de gobierno a consumidor por ejemplo pagos de impuestos o multas vehiculares. G2B o government to business: negocio entre el gobierno y las empresas como los portales de compras y licitaciones. (pág.7)

Gracias a la utilización de la tecnología la forma de comercializar ha experimentado buenos cambios generacionales, en la actualidad la incursión en el e-commerce permite aprovechar los beneficios que ofrece la globalización (Meltzer, 2018). La importancia del e-commerce tiene un marcado crecimiento a través de los años por lo que, actualmente, comercializar productos a través de esta modalidad representa un paso adelante respecto a la competencia.

Fomentar el uso de las TIC en el comercio, a nivel doméstico y en el ámbito de los negocios internacionales en Ecuador es considerado como un desafío. Algunas instituciones como la Cámara Ecuatoriana de Comercio Electrónico (CECE) en conjunto con el E-commerce Institute, han efectuado eventos como el denominado $\boldsymbol{e}$ commerce Day Ecuador, el cual se realiza también en otros países, con el objetivo de divulgar la importancia y beneficios que brinda la economía digital a nivel de empresas, creando de esta manera cierto nivel de reflexión en relación al poder e impacto que representan la internet y las nuevas tecnologías en el mercado actual ( $E$ commerce Day, 2018). Los resultados, han sido llevar al país a ocupar el quinto puesto a nivel de Sur América en los países con mayor uso de red.

Las estadísticas de la Superintendencia de Bancos (2019) reflejan los registros positivos que Ecuador ha mostrado en los últimos periodos con actividades vinculadas al comercio electrónico, en el mejor de los escenarios están las categorías categoría C2C o B2C que por lo general se tratan de operaciones comerciales entre consumidores o entre empresa y consumidor final. En la tabla 1 se detalla la actividad que ha tenido Ecuador en el comercio electrónico, tanto en portales nacionales como internacionales.

Tabla 1

E-commerce en Ecuador

\begin{tabular}{|c|c|c|}
\hline \multirow[b]{2}{*}{ Año } & Porcentaje de & Porcentaje de compra \\
\hline & $\begin{array}{l}\text { compra- venta en } \\
\text { portales nacionales }\end{array}$ & $\begin{array}{l}\text { en portales } \\
\text { extranjeros }\end{array}$ \\
\hline 2016 & $16 \%$ & $83 \%$ \\
\hline 2017 & $13 \%$ & $87 \%$ \\
\hline 2018 & $13 \%$ & $88 \%$ \\
\hline 2019 & $11 \%$ & $89 \%$ \\
\hline
\end{tabular}

Fuente: Adaptado de observatorio de e-commerce de la Universidad Espíritu Santo (2019)

Partiendo de la información presentada en la tabla 1, se evidencia que el e-commerce de Ecuador gira entorno a compras en portales extranjeros. Las tipologías de comercio electrónico que más usa Ecuador ayudan al fortalecimiento de un sistema comercial basado en las TICS, es muy poco probable que generen beneficios 
directos a nivel de país, ya que en dichas categorías generalmente intervienen mercancías importadas, desaprovechando la oportunidad de manejar el e-commerce como herramienta orientada a la exportación de productos nacionales. (Pesántez Calva, Romero Correa, y González Illescas, 2019, pág. 82)

\section{Gráfico 1}

Panorama del e-commerce en Sudamérica

(Ingreso de venta anual)

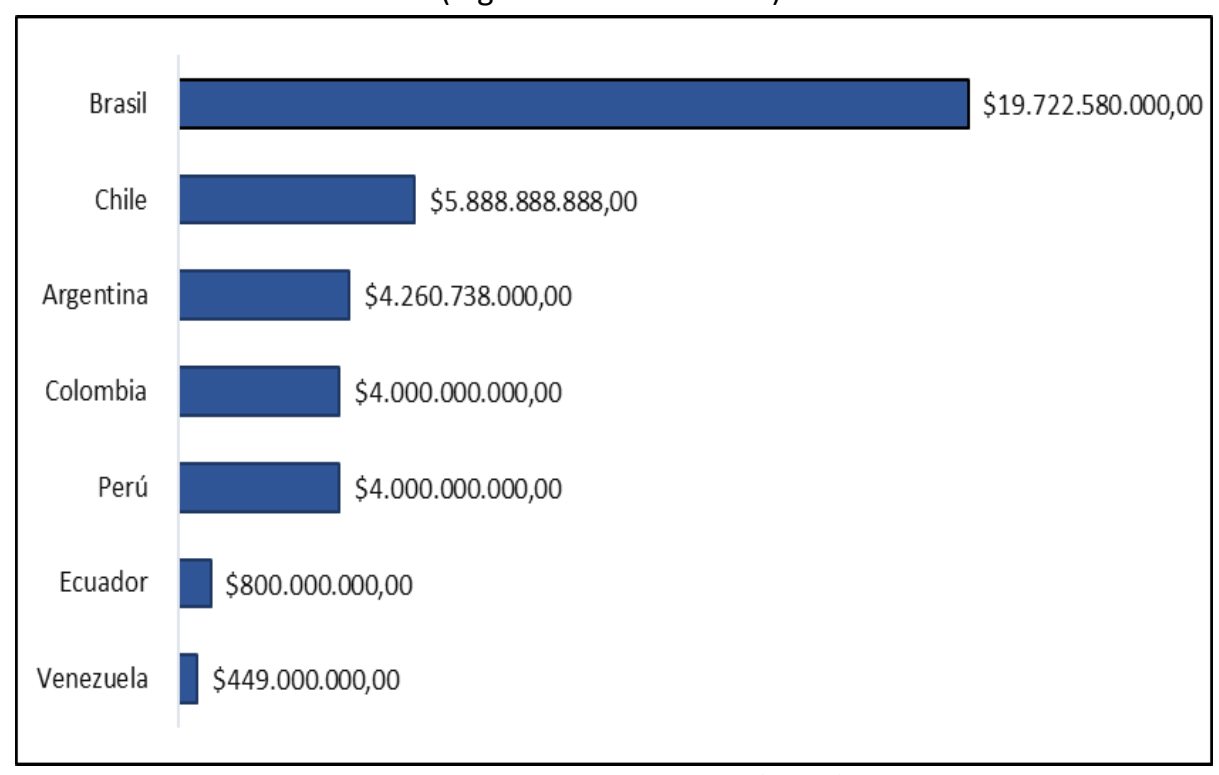

Fuente: Adaptado de Linio (2019)

Estas cifras son bastante alentadoras, que seguramente prometen un futuro muy positivo para las economías de los países que están inmersos en esta nueva modalidad de hacer negocios online y gracias a la continua contribución de la tecnología facilitará la incorporación de nuevas empresas y emprendimientos a un mercado globalizado, logrando convertirse en países más productivos y competitivos.

El e-commerce durante la pandemia ha jugado un papel central en la reducción de brechas tanto del lado de la demanda como la oferta. Esta centralidad del comercio electrónico y el "enfoque digital" llegó para quedarse, ya que consideramos un enfoque doble en "Vidas y medios de subsistencia". La crisis del Covid-19 está impulsando a más consumidores a comprar en línea por lo que la adopción del e-commerce debe de acelerarse; las Mipymes requerirán de mayor adaptación digital.

Al ser este agente un segmento importante en el desarrollo de un país, deben de ser innovadoras y adaptarse a los nuevos cambios, es por esto que, los expertos consideran el e-commerce como una mina de oro para emprender. Schumpeter en sus investigaciones destaca la importancia vital del emprendedor en los negocios, pone énfasis en el emprendedor como innovador y por lo tanto como catalizador de progreso económico.

La relevancia del empresario innovador y del emprendimiento desde el punto de vista del crecimiento económico es evidente. Las innovaciones constituyen un elemento fundamental dentro del funcionamiento de un sistema económico, implican llevar a cabo nuevas combinaciones de factores y que solo un emprendedor innovador puede realzarlas. Schumpeter (1934) argumenta que:

Las actividades de innovación que solo son realizadas por emprendedores involucran las siguientes: 1) La introducción de un producto nuevo (o de un producto conocido de una calidad distinta); 2) La introducción de un nuevo método de producción o de comercialización de un producto; 3) La apertura de nuevos mercados; 4) La obtención de nuevos oferentes de materias primas; y 5) La reorganización de una 
industria, lo que puede incluir la monopolización de la referida industria o la destrucción de un monopolio. $(p, 66)$

A partir de estas observaciones se manifiesta que para Schumpeter el proceso innovador es fundamentalmente distinto de un proceso de invención. Las combinaciones llevadas a cabo por empresarios se basan en elementos que de alguna forma ya existen pero que son organizados en una forma novedosa y original. Hoy en día estamos viviendo una era digital y más con la presencia del Covid-19, a raíz de esta situación las Mipymes se ven en la necesidad de reinventarse dejando de lado al comercio tradicional por el e-commerce.

De ahí se desprenden, las habilidades gerenciales, ya lo decía Schumpeter, quien presenta la característica de liderazgo como un estricto rigor que conduce a la innovación en la economía. El liderazgo requiere de una efectiva comunicación, esta comunicación entre líder y equipo permite que todo fluya de forma natural. Al respecto Parrales Poveda, Gracia Panta, Miller Zavala y Ponce Cedeño (2017) mencionan que:

Saber comunicar puede contribuir a incrementar las competencias de las empresas e instituciones, porque éstas, constantemente, se retroalimentan de las críticas y sugerencias de los empleados, clientes, proveedores, entre otros, quienes, al ser atendidos por el nivel ejecutivo, sin restricciones, se sienten motivados y comprometidos con su evolución, a través del sentido de pertenencia y de un clima de trabajo integrador. (p.10)

El e-commerce es la clave para que empresas como las Mipymes sigan en el mercado, solo aquellas que sean capaces de adaptarse, mejorar procesos y fortalecer conocimiento, además de lograr el compromiso de sus colaboradores y el reconocimiento del mercado, serán las que logren remontar su curva de crecimiento con mayor velocidad, ganándole y a la vez combatiendo la crisis que genera una pandemia.

Encontrarse con tiendas virtuales o empresas con presencia en la web, no es un tema nuevo para Ecuador, ya que se estima que alrededor de 700 tiendas online, existen en el país (El Comercio, 2019). Este escenario es muy distinto al identificar instituciones que brinden servicios orientados al desarrollo de negocios en línea, en donde se evidencia una escasa existencia.

\subsection{Plataformas del e-commerce}

Las ventas por internet se realizan mediante plataformas de comercio electrónico, mediante estas plataformas se pueden efectuar compras de manera segura por medio de internet. Lancetalent (2014) menciona que las empresas utilizan las plataformas en función de las características y herramientas del negocio, según el autor dentro de las plataformas hay algunas que son gratuitas y pagadas, a continuación, se revisan:

- PretaShop: Es una herramienta sencilla de administrar, muy intuitiva, con información relevante muy accesible y que ofrece una buena experiencia de usuario, tiene una interface muy amigable y orientada a facilitar la compras, muy moderna y con posibilidades de personalización. La administración e instalación es muy sencilla, permite la interactividad y usabilidad del usuario. Una característica interesante es que viene en 65 idiomas.

- Magento: Es una herramienta más compleja de instalar, pensada más a personas que tengan conocimientos para resolver problemas técnicos de nivel medio o avanzado. Es la más utilizada, incluso por empresas de gran tamaño.

- VirtueMart: Es una completa solución de e-commerce que puede utilizarse tanto para una tienda on-line como para un catálogo on-line. VirtueMart se trata de una extensión del conocido CMS Joomla un potente gestor de contenidos que permite desarrollar sitios web dinámicos e interactivos totalmente personalizables. 
- Wix: Esta es una solución ideal para principiantes. A través de ella, cualquiera puede desarrollar un e-commerce de forma gratuita. Simplemente hay que elegir entre una de las plantillas que ofrece y seguir los pasos que indica la plataforma.

- Shopify: Es una plataforma muy interesante y fácil de utilizar. No se necesita tener grandes conocimientos informáticos para ponerla en marcha y se puede elegir una amplia lista de diseños web con estilos diferentes según el e-commerce.

En la tabla 2 se detallan las instituciones y empresas más conocidas que brindan los servicios antes mencionados en Ecuador:

Tabla 2

Instituciones y empresas vinculadas al

desarrollo de e-commerce en Ecuador

\begin{tabular}{|c|c|c|c|c|}
\hline $\begin{array}{l}\text { Nombre de } \\
\text { institución o } \\
\text { empresa }\end{array}$ & Sector & Actividad & Ventajas & Desventajas \\
\hline $\begin{array}{l}\text { Cámara } \\
\text { Ecuatoriana de } \\
\text { Comercio } \\
\text { Electrónico }\end{array}$ & Privado & $\begin{array}{l}\text { *Ofrecer } \\
\text { capacitaciones, sellos } \\
\text { de confianza. } \\
\text { *Desarrollar negocios } \\
\text { en línea }\end{array}$ & $\begin{array}{l}\text { *Respaldo de asociación } \\
\text { ecuatoriana de software } \\
\text { *Asistencia en } \\
\text { búsqueda de apoyo por } \\
\text { parte del gobierno } \\
\text { *Aliado de la cámara de } \\
\text { comercio de Quito }\end{array}$ & $\begin{array}{l}\text { *Algunas de } \\
\text { sus actividades } \\
\text { no se } \\
\text { encuentran } \\
\text { disponibles. }\end{array}$ \\
\hline Negos Holding & Privado & $\begin{array}{l}\text { *Terminales de } \\
\text { puntos de venta, } \\
\text { facturación } \\
\text { electrónica y botones } \\
\text { de pago } \\
\text { *Asesorías en } \\
\text { estrategias de ventas } \\
\text { electrónicas }\end{array}$ & $\begin{array}{l}\text { *Asesorías y } \\
\text { seguimiento de ventas } \\
\text { *Apoyo en logística } \\
\text { *Respaldo de asociación } \\
\text { ecuatoriana de software }\end{array}$ & $\begin{array}{l}\text { *No cuentan } \\
\text { con apoyo de }\end{array}$ \\
\hline E-cosulting $360^{\circ}$ & Privado & $\begin{array}{l}\text { *Desarrollo Web } \\
\text { *Consultora en } \\
\text { Marketing Digital }\end{array}$ & $\begin{array}{l}\text { * Respaldo de Cámara } \\
\text { ecuatoriana de } \\
\text { Comercio Electrónico }\end{array}$ & $\begin{array}{l}\text { un organismo } \\
\text { público }\end{array}$ \\
\hline
\end{tabular}

Fuente: Revista INNOVA Research Journal (2020)

Finalmente, analizando brevemente la información expuesta en párrafos anteriores, hasta cierto punto se podría considerar que el e-commerce en territorio ecuatoriano, se viene desarrollando de una manera aceptable. A nivel nacional, existen empresas que brindan servicios relacionados a la creación de tiendas virtuales, sin embargo, no han sido consideradas en la tabla anterior, ya que sus actividades se limitan al desarrollo de un sitio web. (Pesántez Calva et al., 2019)

\section{3. ¿Qué ofrece el e-commerce a la Mipymes?}

El comercio electrónico es una herramienta potente para las Mipymes que les permite avanzar rápidamente por las fases de desarrollo. Las Mipymes que innovan de acuerdo al constante avance de las tecnologías son las que logran un impacto en la competitividad y el desarrollo. 
Según consideraciones de Rodríguez Merino ( 2015) algunos de los beneficios que se descantan para este grupo de agentes económicos son: Eficacia de la empresa al simplificar procesos, aumento de ventas al abrir mercados a los que antes no podía acceder, facilita las relaciones entre empresas al reducir procedimientos de interacción entre las mismas, en la cadena de distribución reduce y elimina los intermediarios, reduce costos al eliminar parte de material impreso como catálogos, manuales de servicio y operación, reduce el ciclo de producción creando mercados más competitivos, permite ofrecer técnicas de marketing personalizado, disponibilidad 24 horas al día, todos los días y mayor cercanía con el cliente.

El e-commerce tiene una serie de ventajas respecto al comercio tradicional, pero también tiene algunas desventajas entre ellas destacan: Necesidad de acuerdos internacionales que permitan armonizar la legislación del comercio electrónico, validez legal de los contratos y transacciones en papel, la existencia de control de las transacciones internacionales como el pago y el cobro de impuestos, protección de los derechos de propiedad intelectual y la seguridad de los medios de pagos electrónicos. (Rodríguez Merino, 2015)

Por otro lado, al comprador le proporciona amplias opciones de compra, este puede considerar una diversidad de productos y servicios de un número amplio de vendedores, puede comprar productos que no se encuentran en su localidad y además reduce y ahorra costos y tiempo. Por otra parte, algunas desventajas para los compradores son: desconfianza en los medios de pago, suspicacia al proporcionar información de datos personales al adquirir un producto, problemas con el idioma que utilizan las diferentes páginas electrónicas, ya que la mayoría usa el inglés y pocas el español, existencia de demoras de entrega de los productos que se adquieren por internet e incluso se corre el riesgo de que estos no lleguen o lleguen con defectos de lo que los vendedores no se hacen cargo.

Sin embargo, son más las ventajas que desventajas, razón por la cual muchos negocios han migrado al ecommerce durante esta emergencia, las diferentes Mipymes decidieron adaptarse a este modelo de negocio, entre estas citamos a empresas internacionales y ecuatorianas como: KAO, Pintulac, Adidas, Bebemundo, Novicompu, Medias Roland, Mundo Mágico de Mascotas, Gloria Saltos, Montero, Las Fragancias, L'Occitane, Movistar, Watch-out, Totto, Pacari, Super Paco, entre otras más, apoyadas través de la plataforma Rappi, ya notaron una manera de reinventarse y seguir con sus negocios. (Diario El Mercurio, 2020)

A nivel nacional una de las empresas pioneras en adoptar el e-commerce es la cadena de tiendas por departamento, De Prati, empresa especializada en moda hogar, belleza y tecnología que incursionó en el $e$ commerce en 2007. Su presencia virtual le ha permitido disponibilidad las 24 horas del día. Mediante una compra segura, los usuarios pueden pagar a través del Crédito Directo De Prati, tarjetas de crédito y efectivo. Los cambios y devoluciones son sin costo y tienen envoltura de regalo. Esta, junto con Almacenes Pycca y Créditos económicos dentro de las tiendas favoritas solicitadas para las compras nacionales. (Villafuerte Sornoza, 2020, p.78)

Según datos ofrecidos por la Cámara Ecuatoriana de Comercio Electrónico, ante la presente crisis sanitaria que atraviesa el país la mayoría de consumidores ecuatorianos realizaban compras físicamente y solo un $5 \%$ lo hacían ocasionalmente de manera virtual, adicional a esto informa que también se han incrementados las compras por internet por medio del e-commerce, recalcando que las personas solo lo hacen por necesidad mas no por preferencias. (Diario El Mercurio, 2020)

El e-commerce es una tendencia que está ayudando a superar la crisis por la pandemia, permitiendo desarrollar las actividades comerciales, reactivando el aparato productivo-económico del Ecuador. Consigo trae una serie de oportunidades para las Mipymes a pesar de los duros momentos que vive la sociedad.

El contexto del e-commerce en gran medida puede contribuir a la reestructuración de las empresas, puede ayudar a las Mipymes a optimizar la asignación de recursos al permitir la comercialización, el almacenamiento y el acceso al mercado. El e-commerce, además abre la posibilidad de mayores márgenes de ganancia al bridar 
eficiencias en las cadenas de valor. En los actuales momentos sin precedentes a la Mipymes le es de vital importancia, ya que el comportamiento del consumidor está cambiando debido al entorno macroeconómico, este busca más valor, esta metodología de compra-venta podría contribuir en gran medida a reestablecer la economía y mantener en funcionamiento el motor consumo.

\subsection{Crecimiento del E-commerce durante la pandemia del Covid-19}

En lo que va de la emergencia sanitaria el sector productivo opera en un $20 \%$ de su capacidad en la industria, comercio y exportaciones. El Covid-19 afectó directamente a las empresas, el sector productivo no resiste sin producir, la falta de liquidez está provocando que los negocios pequeños quiebren y que los grandes empiecen a tambalearse. Analizando la situación económica del país desde una óptica médica el Covid-19 complicó enfermos que ya estaban muy graves en Ecuador, las empresas y el empleo, si bien en el 2019 hubo el mayor número de compañías inscritas casi 10.000 también hubo el mayor número de cierre desde el 2016. (Politicamente correcto, 2020)

Por ello, Ecuador recibió préstamo para apoyar a las Mipymes por 260 millones de dólares, fue aprobado por el Directorio Ejecutivo del Banco Mundial para la Corporación Financiera Nacional (CFN). Esta inyección de capital busca promover el acceso a financiamiento con fines productivos para las Mipymes ecuatorianas, apoyando así su reactivación y recuperación económica, tras las grandes pérdidas que deja el Covid-19. Y a partir de ello ver oportunidades para la sociedad, generando fuentes de empleos y que las empresas no cierren hasta reactivar la economía del país, adaptándose a las nuevas tendencias digitales. (América economía, 2020)

Ecuador hasta finales del año 2019 a nivel nacional tenía un crecimiento lento, y es que, los esfuerzos para sumarse a la tendencia global del e-commerce llevan varios años y se ha acentuado en estos últimos meses. La no existencia de una cultura tecnológica desarrollada en el país, había impedido que muchas empresas se adapten a los cambios digitales que presenta el mercado actual. A causa de la pandemia y el deseo de supervivencia de las Mipymes el e-commerce se ha intensificado, aunque de manera forzosa. Existen empresas que son renuentes al cambio y esto se traduce en la lógica de que algunas empresas ecuatorianas muestren desconfianza a sistemas basados en el e-commerce, muchas veces ocasionados por el desconocimiento o por el simple temor de enfrentarse a nuevos retos, desaprovechando así la tecnología y atender nuevas oportunidades, que las tendencias actuales ofrecen.

Las compras en linea ganan terreno en Ecuador, en 2019 el comerico electrónico movió unos UDS 1.600 millones, y se preveía que en 2020 esta cifra creciera en un 40\%, pero en la cuarentena por la emergencia sanitaria, solo entre el mes de marzo y mayo se movieron alrededor de UDS 500 millones. Esto es, casi un tercio de todo lo que se vendió el año pasado, y se espera que las ventas por esta vía lleguen a los UDS 2.200 millones este año. (EI Comercio, 2020)

\subsection{Impacto del Covid-19 en el e-commerce en Ecuador}

El impacto de la enfermedad por el Covid-19 ocasionó un hipercrecimiento del e-commerce en la industria, debido al cierre de espacios físicos, provocando tanto a nivel de usuario como a nivel de consumo un volcamiento a este canal. Sectores como el de entretenimiento, turismo, aviación, entre otros eran los grandes sectores que marcaban el comercio electrónico, ahora el consumo se volvió bastante esencial, el crecimiento de todo lo que tiene que ver con alimentos, salud e higiene ha sido muy grande.

Al paso del tiempo se han podido observar diferentes tipos de medios y canales de compras, alguno de los canales que más destacan son las páginas web, la mensajería instantánea como WhatsApp, redes sociales como Instagram o Facebook, aplicaciones móviles, portales de e-commerce como mercado libre y otros tomando en cuenta que el mayor porcentaje de utilización corresponden a la mensajería instantánea como WhatsApp con 
un $49 \%$ y las aplicaciones móviles con un $44 \%$. La principal categoría de productos adquiridos por medio de estos canales se da principalmente en víveres y comidas preparadas, salud, tecnología y educación, teniendo así los alimentos y bebidas no alcohólicas en primer lugar con un $67 \%$ el segundo lugar la salud y medicinas con un $50 \%$, en tercer lugar, los restaurantes o las comidas preparadas, la tecnología con un 19\% y los servicios de educación o capacitación un $15 \%$. (CECE, 2020)

Existen diversos tipos de experiencias con las compras en línea durante la enfermedad por Covid-19, muchas personas en su mayoría señalan que las compras por medio del internet han sido muy útiles en este momento, les ha permitido comprar y pagar desde la comodidad de su casa. Entre sus ventajas el e-commerce ofrece facilidad de pago, desde tiempo atrás se ha tenido conocimientos de qué existen diversas formas de pagos que se pueden realizar por diferentes medios cuando se habla de la compra-venta por internet, algunos de ellos son la tarjeta de crédito, la tarjeta de débito, el efectivo y las transacciones bancarias. Sin embargo, con la presente pandemia se han adoptado medios de pagos alternativos como lo son la billetera electrónica, el Bitcoin y el código QR los cuales han tenido una mayor aceptación entre los más jóvenes y las personas de menores ingresos. Sin embargo, algunas personas muestran una postura no tan positiva frente a las transacciones realizadas a través del e-commerce, catalogándolas como decepcionantes y complicadas.

El Covid-19 ha traído cambios de hábitos importantes, tanto por el aislamiento obligatorio como por el voluntario con consecuencias en las cadenas de abastecimiento, sin embargo, esto lleva a satisfacer necesidades mediante nuevas modalidades como son el uso del internet y las copras en línea. A pesar de las comodidades que el $e$ commerce brinda se provee que el canal tradicional se vea afectado después de la pandemia cuando no haya necesidad de aislarse, ya que las personas estarán muy concentradas en las compras por internet. Según la Cámara de Comercio Electrónica Ecuatoriana (2020) se tiene previsto que un 32\% de las personas seguirán comprando por internet, un $11 \%$ aumentarán sus compras por internet, el $17 \%$ volverá a la cotidianidad de ir a la tienda física frecuentemente y un $40 \%$ irá a la tienda física, pero con menor regularidad.

A corto plazo, el e-commerce en Ecuador ha permito a las Mipymes mantener en funcionamiento sus cadenas de suministro evitando el contacto en persona. A mediano y largo plazo se estima que el e-commerce ayudará a efectuar transacciones más allá de las fronteras de los estados y países al ofrecer plataformas a las que se puede acceder en todas las geografías. Esta facilidad de acceso es fundamental a la luz de la escasez de liquidez y la disminución de la capacidad financiera de compradores y productores.

Atendiendo a estas consideraciones y dado que las empresas tardarán algún tiempo en volver al ritmo normal de una era anterior a Covid-19, las Mipymes deben de garantizar su supervivencia. A la luz de esto, ha llegado el momento de que el sector de las Mipymes adopte la tecnología digital más rápidamente que nunca. El comercio electrónico tiene el potencial de impulsar este sector empresarial, y la capacidad de digitalizar de manera oportuna será un diferenciador significativo entre las organizaciones a medida que se transforman para adaptarse a las necesidades cambiantes del momento y a las nuevas tendencias sociales.

\section{Metodología}

El presente trabajo tiene un diseño de tipo documental, se hace uso de métodos científicos teóricos y empíricos. Se ha recurrido a información de fuentes directas e indirectas, partiendo de un orden cronológico se revisaron bases de datos académicas-científicas, mediante la búsqueda de palabras clave relacionadas con el tema de investigación (Covid-19, Mipymes, e-commerce) a través de las cuales se recopiló un cúmulo de documentos relacionadas con las variables de estudio, esto permitió analizar, estudiar y seleccionar la información útil de otros investigadores que conformaría parte de la redacción del content matter. 
La búsqueda de información se hizo a partir de fuentes primarias como artículos, tesis, informes técnicos, folletos, memorias de ponencias, las cuales se seleccionaron de acuerdo a la pertinencia con el tema de estudio, además se hizo una revisión de fuentes físicas como libros y periódicos donde se recopiló conceptos y opiniones importantes de otros investigadores para fundamentar la investigación.

Entre los métodos teóricos se utiliza el histórico y lógico, para revelar la evolución de las transacciones de compra-venta en los últimos años y cómo las Mipymes lo han venido implementando, más aún en tiempos de Covid-19.

Los métodos de carácter empírico se enmarcan en la observación, a partir de la realidad y de la propia experiencia sobre el tema del e-commerce, lo que permitió observar cómo usuarios y Mipymes emigran al e-commerce para seguir ofertando y demandando en tiempos de Covid-19, tomándolo como el único salvavidas debido a las medidas de confinamientos por la pandemia.

\section{Resultados}

Ecuador es un país de Mipymes, que juegan un papel transcendental en el desarrollo económico del país, aportan significativamente en la generación de empleo y riqueza, representan dentro del tejido empresarial el 99,5\%. Sin embargo, a pesar de ser un grupo mayoritario de agentes económicos son las que más confrontan problemas para sobrevivir en el mercado y alcanzar crecimiento, tales como el limitado acceso al financiamiento, la falta de recursos humanos especializados, subutilización de la capacidad instalada y carencia tecnología, por mencionar algunos de los obstáculos más importantes.

El e-commerce ha ido ganando terreno en los últimos años desde países más desarrollados hasta los que están en vías de desarrollo, a nivel mundial China lidera el volumen de ventas en línea con $\$ 636.087 .000 .000$, seguido Estados Unidos con ventas de \$504.582.000.000. En América latina las ventas en línea han tenido un incremento importante dentro del ranking de e-commerce, Ecuador se encuentra ubicado en el quinto lugar generando ventas anuales de $\$ 800.000 .000$, superado por Brasil, país que lidera la lista con ventas de $\$ 19.722 .580 .000$.

El Covid-19, afectó directamente a las empresas, el confinamiento paralizó cerca del 70\% de las actividades económicas, las más afectadas fueron el área comercial, de servicios, manufactura y agricultura. Es así que en 2020 se tuvo a 432 empresas canceladas entre el mes de marzo y abril, debido a la crisis económica producto de la pandemia, el cierre de sus actividades en parte y la reducción significativa de su producción por otro lado ocasionaron la falta de liquidez haciendo que estas no se mantengan en pie. De las Mipymes ecuatorianas el 75 $\%$ de las microempresas y el $50 \%$ de las medianas y pequeñas empresas solo pueden resistir sin liquidez en promedio 28 días.

La llegada del Covid-19 trajo para este grupo de empresas un cambio dramático en la manera de comercializar productos y servicios, el comercio tradicional pasó a ser un método de segundo plano, y el e-commerce que hasta antes de 2019 no era adoptado por las empresas, paso a ser una de las industrias ganadoras. Las ventas en línea saltaron de 1.200 millones en 2018 a 1.600 millones en 2019 y en 2020 en tiempos de pandemia solo entre el mes de marzo y mayo se movieron alrededor de 500 millones.

La Mipymes que no cerraron, para sobrellevar las restricciones que incluyen el distanciamiento social y el cierre total o parcial de varias industrias y negocios, han considerado como aliada la transformación digital. El Covid-19 causó un incremento en la actividad comercial en línea, es así que las compras por canales digitales o sitios web han incrementado al menos en 15 veces desde el inicio del distanciamiento social, recalcando que antes del confinamiento las personas que usaban plataformas digitales como medios de compra secundario pasaron a usar estas plataformas constantemente, debido al miedo de exponerse al contagio al asistir personalmente a realizar sus compras, por lo que el e-commerce es el medio para evitar salir de casa. 
La llegada de la pandemia del Covid-19 aceleró la adopción de las ventas en línea de grandes compañías, pero también de las Mipymes. Las cifras globales del comercio electrónico son difíciles de cuantificar para un mercado pequeño como el ecuatoriano. A finales de abril, se supo que Amazon presentó unos beneficios por \$2 535 millones entre enero y marzo del 2020. Este monto es cerca del 3\% del producto interno bruto del Ecuador. Sin embargo, esos beneficios del gigante del comercio electrónico son un 29\% menos que los \$ 3561 millones que obtuvo en beneficios el primer trimestre del año pasado. Aun así, la firma se mantiene como referente del comercio electrónico global. Es la vitrina de miles de productos.

Una de las Mipymes ecuatorianas con éxito en el e-commerce es De Prati, esta empresa cuenta con un canal digital hace 12 años, en 2019 las ventas en línea representaron 3\% del total de la facturación, con la actual coyuntura de la emergencia sanitaria, proyectan tener un gran crecimiento a gran escala de doble digito debido al aumento de las visitas que registran por día en su sitio web que muestran un crecimiento por más del $50 \%$. Por otro lado, tenemos a Funky Fish, inició su plataforma e-Commerce hace tres años y vio cómo su promedio de venta que era del $10 \%$ del total, hoy aumentó al $15 \%$.

La pandemia superó uno de los retos dentro de la industria en Ecuador, la digitalización en los negocios, las Mipymes ahora para seguir operando utilizan el teletrabajo, y para ofertar productos adoptaron el e-commerce como nuevo método de comercialización, hoy la mercadotecnia es una tendencia. Los negocios encontraron en el mercado nuevas oportunidades para tener contacto más directo y automatizado con sus clientes, ahora la experiencia digital es única y necesaria para reducir las distancias entre personas y empresas, las empresas ofrecen servicios personalizados, los pedidos son acorde a las necesidades de los clientes, algunas de ellas para no cerrar sus negocios debido a la escasez de demanda diversificaron su producción, negocios como los restaurantes ahora venden los ingredientes para las comidas y mediante tutoriales transmiten a sus clientes como se preparan, otros sectores también se han diversificado, como las empresas que venden bebidas alcohólicas, pasaron a producir mascarillas, guantes, gel antibacterial etc. Todo esto es parte de la innovación de las empresas.

La presión por comprar sin tener contacto con otras personas, por temor a contagiarse y las restricciones para movilizarse en las diferentes ciudades del país llevó a los consumidores a ingresar al canal electrónico. Así lo manifiesta un estudio de Transacciones electrónicas en Ecuador durante el Covid-19 realizado de manera digital a 1125 personas, la última semana de mayo. Los resultados muestran 13 de cada 100 compradores comenzaron a adquirir bienes y servicios de manera digital por primera vez a partir de la pandemia. Otro de los hallazgos del estudio está vinculado al comportamiento de los canales de compra. Los más usados han sido WhatsApp (49\%), aplicaciones de adquisición y delivery (44\%) y páginas web (35\%). La concentración a nivel local referente al ecommerce en Ecuador, ha sido notorio, logrando promover las compras y ventas en portales nacionales e internacionales las cuales se enmarcan en la categoría C2C o B2C.

Las Mipymes siendo un segmento importante dentro de las economías, al ser el mayoritario número de firmas existentes para no quedar rezagadas tienen que innovar constantemente. Schumpeter (1934) en sus estudios de emprendimiento e innovación habla de que las innovaciones constituyen un elemento fundamental dentro del funcionamiento de un sistema económico y las que se llevan a cabo se basan en elementos que ya existen pero que aún no son implementados. La innovación es una de las características de las Mipymes ecuatorianas, quienes en tiempos de Covid-19 han mostrado su capacidad de resiliencia, aquellas que no se adapten a esas tendencias de transformación digital y al e-commerce se verán afectadas con el cierre o ya estarán a punto de cerrar, las empresas tienen que atreverse al desafío, escudriñar nuevas tendencias, observar comportamientos, aprender de experiencias exitosas y desaprender paradigmas qué bloquean el uso de las herramientas digitales. Un ejemplo de empresa virtual exitosa es Picker, dedicada a la entrega de todo tipo de productos por un contacto previo entre usuario y negocio, a través de su App. Inicialmente hace 10 años abrió una plataforma virtual 
denominada despensaenlinea.com para vender productos de un supermercado y una ferretería, el negocio en ese entonces no resultó. Años más tarde conociendo más sobre el modelo de negocio buscó financiamiento con aporte de inversores y hasta con ahorro de la familia, es así que arrancó con el desarrollo de la aplicación, para octubre de 2018 ya tenía plataforma digital y así arranco Picker. Por otro lado, Tipti que opera desde finales del 2017 en Quito y Guayaquil dedicada a ofrecer servicio de asistencia de compras para productos de supermercado, en apenas dos años ha logrado posicionarse entre las mejores del mercado nacional. Ha recibido premios vinculados el comercio electrónico entre los que figuran el reconocimiento a la mejor pequeña y mediana empresa del sector en Ecuador, otorgado en 2019 en los e-commerce Awards. Su éxito es tal, que pasó de facturar menos de UDS 80.000 en el primer año a cerrar en 2019 con ventas superiores a UDS 2 millones. Y en 2020 gracias a ello espera expandir el servicio a otras ciudades.

Las Mipymes para seguir desarrollando sus actividades se vieron en la necesidad de adaptarse a las nuevas tendencias sociales, mejorando procesos, replanteando sus culturas organizacionales y fortaleciendo sus sinergias para remontar la curva de crecimiento con mayor velocidad, lidiando la crisis económica generada por la pandemia del siglo XXI.

\section{Conclusiones}

El Covid-19 ha generado un futuro incierto en varias industrias, pero en el caso del e-commerce generó una oportunidad. No se sabe cuánto durará la pandemia, ni cuál será su efecto en la sociedad, y por esta razón es importante la resiliencia por parte de los negocios. Es momento de adaptarse a la nueva modalidad de vida y cambiar nuestra mentalidad. Es momento de dejar de ver al Covid-19 como una amenaza para las empresas, que implica el cierre o desaparición de las mismas y empezar a verlo como una oportunidad para abrir nuevos canales de negocio, lo cual se puede resumir en una palabra "Reinventarse".

Para alcanzar el éxito los negocios deben de enmarcarse dentro de la versatilidad empresarial para responder a la exigencia de cambios que dependen mucho de algunos factores importantes como la disponibilidad de herramientas tecnológicas, la sinergia desarrollada en los equipos de trabajo y la discusión al cambio en su cultura organizacional.

Es importante tomar esta oportunidad que indirectamente trajo consigo el Covid-19 y hacer frente a otros desafíos que implica la digitalización en los procesos de comercialización de los negocios, tanto el ámbito de seguridad, de manejo y protección de datos y la experiencia del consumidor, de tal manera que al término de la emergencia del e-commerce continúe.

El Ecuador ha venido soportando enormes deficiencias en infraestructura de telecomunicaciones, de cultura y educación para impulsar el crecimiento de los mercados electrónicos, lo que representa una desventaja competitiva en el escenario mundial. Actualmente las insuficiencias van siendo disminuidas, a través de iniciativas aisladas tanto de parte del gobierno Nacional, (a través del desarrollo de proyectos de compras públicas, dinero electrónico, facturación electrónica, entre otros) como del sector empresarial. El e-commerce en Ecuador ha venido creciendo a un ritmo lento, debido a que las Mipymes en su mayoría, por el tamaño de sus estructuras se han abstenido a involucrarse en el mundo de las tendencias en estrategias de negocios, han seguido por décadas un paradigma empresarial, basado en el comercio tradicional, mostrándose reacias al cambio por temor, desconocimiento y sobre todo desconfianza en nuevos métodos comerciales que los negocios traen conforme la dinámica empresarial avanza.

Sin embargo, el Covid-19 a raíz de la suspensión de actividades en un 70\% del sector empresarial logró ese punto de inflexión y activar esa capacidad de adaptación de las Mipymes, aun así, la consolidación del comercio 
electrónico señala un camino medianamente explorado en Ecuador y que se espera en el mediano plazo aumente.

Las políticas públicas que decrete el gobierno son fundamentales para reactivar la economía de las Mipymes, a través de créditos, mediante programas como el Plan reactívate Ecuador y el Crédito Pymes exprés que otorga la Corporación Financiera Nacional (CFN) se ha pretendido ayudar a este sector, sin embargo, resulta difícil acceder al financiamiento, hay muchos obstáculos para estos negocios pequeños. Estas políticas implementadas para ayudar a mejorar su situación financiera y mantenerse en el mercado no están funcionando, muchos negocios se quejan de que los recursos no llegan y el tiempo se agota. Por lo que, el apoyo es fundamental para poder mantener reactivada la economía.

\section{Referencias bibliográficas}

Aguerre, C. (2019). Digital trade in Latin America: mapping issues and approaches. Digital Policy, Regulation and Governance, 21(1), 2-18.

América economía. (Abril de 2020). ¿Cómo enfrentar la crisis en las empresas? Los aliados en cuarentena. Vistazo, 172.

Boletín Juridico de la Cámara de Comercio de Quito. (2017). Clasificación de las Pymes, pequeña y mediana empresa. Recuperado de http://www.ccq.ec/wpcontent/uploads/2017/06/Consulta_Societaria_Junio_2017.pdf

CAN. (s.f.). Mipymes. Recuperado de Comunidad Andina: http://www.comunidadandina.org/Seccion.aspx?id=313\&tipo=TE\&title=mipymes\#: :text=Las\%20micro\%2 C\%20peque\%C3\%B1as\%20y\%20medianas,de\%20las\%20prioridades\%20de\%20la

CECE. (2020). Transacciones electrónicas en Ecuador durante el Covid-19. Recuperado de https://cece.ec/wp-content/uploads/2020/06/Transacciones-electronicas-en-Ecuador-durante-elCovid19.pdf

CEPAL. (2020). América Latina y el Caribe ante la pandemia del enfermedad por coronavirus : Efectos económicos y sociales. Recuperado de https://repositorio.cepal.org/bitstream/handle/11362/45337/S2000264_es.pdf?sequence=6\&isAllowed=y

Ecomerce Day. (2018). Ecommerce Day Ecuador. Recuperado de http// www.ecommerceday.ec/2018/elevento/

El Comercio. (2019, 1 de Abril). El E-commerce duplicó ventas en dos años. Recuperado de https://www.elcomercio.com/actualidad/comercio-electronico-ventas-ecuador-estudio.html

El Comercio. (2020, 29 de Junio). Ventas 'on line' sumarán USD 2200 millones en este año, proyecta gremio. Recuperado de: https://www.elcomercio.com/actualidad/ventas-online-negocios-economiacomercio.html\#: :text=Durante\%20el\%202019\%2C\%20el\%20comercio,se\%20vendi\%C3\%B3\%20el\%20a\%C 3\%B10\%20pasado.

El Mercurio. (2020, 7 de Mayo). Comercio electrónico: una tendencia irreversible. Recuperado de https://ww2.elmercurio.com.ec/2020/05/07/comercio-electronico-una-tendencia-irreversible/

García, J. H. (2018). La regulación del comercio: retos ante el cambio tecnológico. Revista del Instituto de Ciencias Jurídicas de Puebla, 12(41), 43-70. 
Granda Bohórquez, J. P. (2020). Las Pymes y el manejo de los estados financieros en la cuidad de Machala (Tesis). Universidad Técnica de Machala. Machala-El Oro. Recuperado de http://repositorio.utmachala.edu.ec/bitstream/48000/13051/1/ECUACE-2018-CA-DE00927.pdf

INEC. (2016). Directorio de Pymes 2016. Recuperado de INEC: http://www.inec.gob.ec/documentos_varios/presentacion_direc_empresas.pdf

Lancetalent. (26 de Septiembre de 2014). ¿Cuál Es La Mejor Plataforma Para Crear Tu Tienda Online? Recuperado de https://www.lancetalent.com/blog/cual-es-la-mejor-plataforma-crear- tienda-online/

LINIO . (2019). Índice mundial del comercio electrónico . Recuperado de https://www.linio.com.ar/sp/indiceecommerce

Meltzer, J. P. (2018, agosto). A digital trade policy for Latin America and the Caribbean.Banco Interamericano de Desarrollo.

Ministerio de Telecomunicaciones y de la Sociedad de la Información. (2020). El 82,3\% de Mipymes en el Ecuador utilizan Internet. Recuperado de https://www.telecomunicaciones.gob.ec/el-823-de-mipymes-enel-ecuador-utilizan-internet/

Observatorio de Comercio Electrónico de la Universidad Espíritu Santo. (2019). Comportamiento de las transacciones no presenciales en Ecuador 2019. Universidad Espíritu Santo.

OIT (Organización Internacional del Trabajo) (2020). “Enfermedad por coronavirus y el mundo del trabajo: repercusiones y respuestas", 18 de marzo de 2020 [en línea]. Recuperado de:

https://www.ilo.org/wcmsp5/groups/public/-dgreports/dcomm/documents/briefingnote/wcms_739158.pdf.

Parrales Poveda, M., Gracia Panta, E., Miller Zavala, J., \& Ponce Cedeño, O. (2017). Lineamientos Estratégicos y Comunicación Digital (Primera ed.). Mawil Publicaciones de Ecuador.

Parrales, M.L. (2013). Solucionar Problemas Profesionales SPP, una competencia indispensable en la vida laboral y profesional (Primera ed.). Editorial Publish DM. Área de la Educación, sub-área: Formación del Personal Docente y Ciencias de la Educación.

Pesántez Calva, J. A., Romero Correa, J. A. \& González Illescas, M. L. (2020 ). E-commerce B2B como estrategia competitiva en el comercio internacional: Desafíos para Ecuador. INNOVA Research Journal, 5(1), 72-93.

Piñeiro, C. \& Rodríguez, M. (2016). Las TIC como inductores de competitividad y facilitadores del éxito empresarial. International Journal of Information Systems and Software Engineering for Big Companies (IJISEBC), 3(1), 8-26.

Politicamnete correcto. (3 de Mayo de 2020). ¿El desempleo es la próxima pandemia? [Archivo de video]. Recuperado de https://www.youtube.com/watch?v=hJINDB2ypao

Rodriguez Merino, C. (12 de agosto de 2015). ¿Qué es el E-commerce o comercio electrónico?. Blog del Máster en Marketing Directo y Digital de la UPF Barcelona School of Management. Recuperado de http://marketingdigital.bsm.upf.edu/e-commerce-comercio-electronico/

Rodriguez Valencia, J. (2007). Administración de pequeñas y medianas empresas (Quinta ed.). México D.F: Thomsom. 
Ron Amores, R. \& Sacoto Castillo, V. (2017, Julio 28). Las PYMES ecuatorianas: su impacto en el empleo como contribución del PIB PYMES al PIB total. Revista Espacios . Recuperado de https://www.revistaespacios.com/a17v38n53/a17v38n53p15.pdf

Schumpeter, J. A. (1934). The Theory of Economic Development. Cambridge, Mass: Harvard University Press.

Seoane , E. (2005). La nueva era del comercio electrónio: El comercio electrónico. Las TIC al servicio de la gestión empresarial. (Primera ed.). Madrid, España: Vigo:Ideaspropias. Recuperado de https://books.google.com.ec/books?id=evLz521ZVmAC\&printsec=frontcover\&hl=es\&source=gbs_ge_sum mary_r\&cad=0\#v=onepage\&q\&f=false

Sornoza Parrales , G., Parrales Poveda , M., Sornoza Parrales , G., Cañarte Rodriguez , T., Castillo Merino , M., Guaranda Sornoza, V., \& Delgado Lucas, H. (2018). Fundamenos de emprendiemiento (Primera ed.). Editorial Área de Innovación y Desarrollo,S.L.

Superintendencia de Bancos. (2018). Portal estadístico Superintendencia de Bancos. Recuperado de http://estadisticas.superbancos.gob.ec/portalestadistico/portalestudios/?page_id=1826

Tello Pérez, P. \& Pineda González , L. (2017). Análisis del comercio electronico en el Ecuador (Tesis de maestría). Universidad Internacional del Ecuador. Quito-Ecuador. Recuperado de https://repositorio.uide.edu.ec/bitstream/37000/2476/1/T-UIDE-1768.pdf

Villafuerte Sornoza, S. (2020). Las compras on-line y el comportamiento del consumidor de la ciudad de Jipijapa (Tesis). Universidad Estatal del Sur de Manabí. Jípijapa-Manabí.

Zapata, C. (2020, 11 de Mayo). Oportunidades de negocios se multiplican con el e-commerce. Líderes. Recuperado de https://www.revistalideres.ec/lideres/revista-lideres-edicion-11-mayo.html.

Esta obra está bajo una Licencia Creative Commons Attribución-NoCommercial 4.0 International

\section{(cc) BY-NC}

